\title{
Errata
}

\section{Vol. 67, No. 9}

In the report "Update: Dura Mater Graft-Associated Creutzfeldt-Jakob Disease - Japan, 1975-2017," on page 276, an error occurred in Figure 3. The corrected figure is as follows:

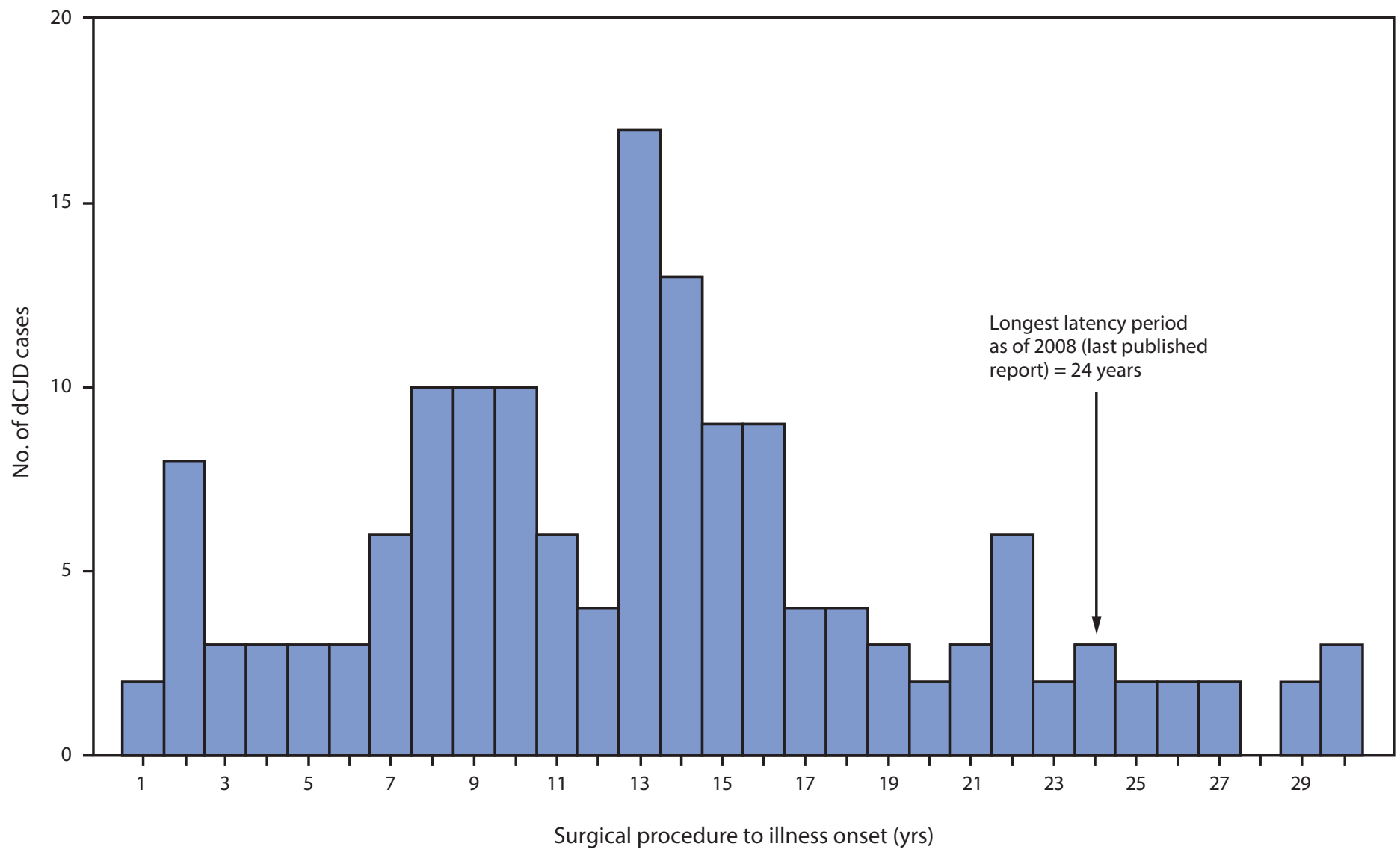

\section{Vol. 67, No. 7}

In the report "HIV Diagnoses Among Persons Aged 13-29 Years - United States, 2010-2014," on page 212, the second footnote for Table 1 should have read "† Rates are single-year rates per 100,000 population. Rates for 2010-2014 are comparable to single-year rates and are calculated as number of diagnoses during 2010-2014 divided by number of person-years at risk for diagnosis during 2010-2014 (x 100,000)." On page 213, the third footnote for Table 2 should have read " $\$$ Rates are 5-year cumulative rates per 100,000 population. Rates are not calculated by transmission category because of the lack of denominator data." 